\title{
Fatores de risco cardiovascular e qualidade de vida de estudantes de enfermagem*
}

Cardiovascular risk factors and quality of life of nursing students

Factores de riesgo cardiovascular y calidad de vida de estudiantes de enfermería

\section{Daiana Cristina Wickert ${ }^{\mathrm{I}}$, Laís Mara Caetano da Silva ${ }^{\mathrm{II}}$, Oclaris Lopes Munhoz ${ }^{\mathrm{III}}$, Maria Denise Schimith $^{\mathrm{IV}}$, Tânia Solange Bosi de Souza Magnagov ${ }^{\mathrm{v}}$, Vanessa do Nascimento Silveira ${ }^{\mathrm{VI}}$}

\begin{abstract}
Resumo: Objetivo: identificar os fatores de risco cardiovascular e a percepção da qualidade de vida entre estudantes de enfermagem de uma universidade federal do Rio Grande do Sul, Brasil. Método: estudo transversal descritivo, realizado com 131 estudantes de enfermagem, em 2018. Os dados foram coletados por meio de instrumento de caracterização demográfica e socioeconômica, formulário de identificação de fatores de risco cardiovascular e do questionário The World Health Organization Quality of Life, bref (WHOQOL-BREF). Para análise dos dados empregou-se estatística descritiva. Resultados: evidenciou-se que $115(87,8 \%)$ estudantes apresentavam fatores de risco cardiovascular. Quanto à qualidade de vida, a média dos escores foi de $70,1( \pm 17,0)$ para as relações sociais e 57,8 $( \pm 13,8)$ para o meio ambiente. Conclusão: constatou-se alta prevalência de fatores de risco cardiovascular, prevalecendo o sedentarismo e obesidade. Quanto à qualidade de vida, a pior percepção relacionou-se ao meio ambiente, já as relações sociais evidenciaram resultados positivos
\end{abstract}

Descritores: Sistema Cardiovascular; Qualidade de Vida; Enfermagem; Estudantes de Enfermagem; Saúde do Estudante

\footnotetext{
${ }^{\text {I }}$ Enfermeira. Mestranda em Enfermagem pelo Programa de Pós-Graduação em Enfermagem/UFSM- Bolsista CAPES. Universidade Federal de Santa Maria. Santa Maria, RS, Brasil. E-mail: daianacristinaw@gmail.com - ORCID: http://orcid.org/0000-0001-7180-1428

II Enfermeira. Pós-doutora em Enfermagem em Saúde Pública/EERP/USP. Professora Adjunta do Departamento de Enfermagem e do Programa de Pós-Graduação em Enfermagem/UFSM. Universidade Federal de Santa Maria. Santa Maria, RS, Brasil. E-mail: lais.silva@ufsm.br- ORCID: http://orcid.org/0000-0001-7596-2333

III Enfermeiro. Mestre em Enfermagem/UFSM. Doutorando em Enfermagem pelo Programa de Pós-Graduação em Enfermagem/UFSM. Professor Substituto do Departamento de Enfermagem/UFSM. Universidade Federal de Santa Maria. Santa Maria, RS, Brasil. E-mail: oclaris_munhoz@hotmail.com- ORCID: http://orcid.org/0000-0001-8901-7148

IV Enfermeira. Pós-doutora em Enfermagem/FURG. Professora Adjunta do Departamento de Enfermagem e do Programa de Pós-Graduação em Enfermagem/UFSM. Universidade Federal de Santa Maria. Santa Maria- RS, Brasil. E-mail: maria-denise-schimith@ufsm.br- ORCID: http://orcid.org/0000-0002-4867-4990

V Enfermeira. Doutora em Enfermagem/EEAN-UfRJ. Professora Adjunta do Departamento de Enfermagem e do Programa de Pós-Graduação em Enfermagem/UFSM. Universidade Federal de Santa Maria. Santa Maria- RS, Brasil. E-mail: magnago.tania@gmail.com- ORCID: http://orcid.org/0000-0002-5308-1604

VI Enfermeira. Mestre em Enfermagem/UFSM. Residente em urgência e emergência/UFN. Universidade Federal de Santa Maria. Santa MariaRS, Brasil. E-mail: vanessa.sylveira@hotmail.com- ORCID: http://orcid.org/0000-0001-6289-8977

* Extraído do trabalho de conclusão de curso "Relação entre fatores de risco cardiovascular e qualidade de vida de acadêmicos de enfermagem", Curso de graduação em enfermagem, Universidade Federal de Santa Maria, 2018.
} 
Fatores de risco cardiovascular e qualidade de vida de estudantes de enfermagem | 2

\begin{abstract}
Objective: to identify cardiovascular risk factors and the perception of quality of life among nursing students from a federal university in Rio Grande do Sul, Brazil. Method: descriptive cross-sectional study, conducted with 131 nursing students, in 2018. Data were collected through an instrument for demographic and socioeconomic characterization, a form for identifying cardiovascular risk factors and the World Health Organization Quality of Life questionnaire, Bref (WHOQOL-BREF). In order to analyze data, we used descriptive statistics. Results: we found that $115(87.8 \%)$ students had cardiovascular risk factors. As for quality of life, the average score was $70.1( \pm 17.0)$ for social relationships and $57.8( \pm 13.8)$ for the environment. Conclusion: there was a high prevalence of cardiovascular risk factors, with a prevalence of sedentariness and obesity. Still regarding the quality of life, the worst perception was related to the environment, while social relationships highlighted positive results.
\end{abstract}

Descriptors: Cardiovascular System; Quality of Life; Nursing; Students, nursing; Student Health

Resumen: Objetivo: identificar los factores de riesgo cardiovascular y la percepción de la calidad de vida en estudiantes de enfermería de una universidad federal de Rio Grande do Sul, Brasil. Método: estudio descriptivo transversal, efectuado con 131 estudiantes de enfermería, en 2018. Los datos se recolectaron mediante un instrumento de caracterización demográfica y socioeconómica, un formulario para identificar factores de riesgo cardiovascular y el cuestionario World Health Organization Quality of Life, Bref. (WHOQOL-BREF). Para analizar los datos, se utilizó estadística descriptiva. Resultados: se evidenció que $115(87,8 \%)$ estudiantes tenían factores de riesgo cardiovascular. En cuanto a la calidad de vida, el promedio de puntuación fue de $70,1( \pm 17,0)$ para las relaciones sociales y $57,8( \pm 13,8)$ para el medio ambiente. Conclusión: se observó una alta prevalencia de factores de riesgo cardiovascular, predominando el sedentarismo y la obesidad. En cuanto a la calidad de vida, la peor percepción estuvo relacionada con el medio ambiente, mientras que las relaciones sociales señalaron resultados positivos.

Descriptores: Sistema Cardiovascular; Calidad de Vida; Enfermería; Estudiantes de Enfermería; Salud del Estudiante

\title{
Introdução
}

As doenças cardiovasculares (DCVs) causam 31\% dos óbitos registrados mundialmente, totalizando 17,7 milhões em 2015. ${ }^{1}$ Elas fazem parte do grupo das Doenças Crônicas Não Transmissíveis (DCNTs), incluindo Hipertensão Arterial Sistêmica (HAS), Diabetes Mellitus (DM) e neoplasias. Nesse sentido, sabe-se que a maioria das mortes por DCNTs são ocasionadas por um conjunto de fatores de risco, destacando-se o tabagismo, alimentação inadequada, inatividade física e consumo de bebidas alcóolicas. ${ }^{1}$

As DCNTs são um dos maiores problemas de saúde pública e causa de óbitos no Brasil, gerando incapacidades e limitações que diminuem a Qualidade de Vida (QV). ${ }^{2}$ A Organização Mundial da Saúde (OMS) define QV como a "percepção do indivíduo de sua posição na vida, no 
3 | Wickert DC, Silva LMC, Munhoz OL, Schimith MD, Magnago TSBS, Silveira VN

contexto da cultura e de sistemas de valores em que vive e em relação aos seus objetivos, expectativas, padrões e preocupações”. ${ }^{3: 1}$

Com isso, a identificação precoce e controle dos Fatores de Risco Cardiovasculares (FRCVs) são práticas essenciais para o manejo das DCVs, que repercutem na promoção da saúde e QV, em especial entre universitários. Nessa parcela da população pode ocorrer baixa QV devido a determinadas características demográficas e socioeconômicas, como a distância da cidade natal até a cidade de estudo e, ainda, associada aos comportamentos de risco para DCV, como o sedentarismo, consumo de álcool e sódio em excesso. ${ }^{4}$

Para sustentar o presente estudo, realizou-se uma busca no Portal Regional da Biblioteca Virtual em Saúde (BVS), em março de 2020, com vistas a identificar estudos que abordassem a QV e os FRCVs entre estudantes de enfermagem. Foram identificadas duas pesquisas e ambas indicaram a necessidade de mudanças de hábitos e cuidados para melhoria da QV, entretanto, não procederam à sua mensuração. ${ }^{5-6}$ Assim, justifica-se o presente estudo devido ao aumento das DCVs na população jovem e seu impacto na QV, bem como a escassez de estudos que abordem a QV e FRCVs entre acadêmicos de enfermagem brasileiros.

O tema estudado situa-se no eixo cinco das linhas de pesquisa contempladas pela agenda de prioridades em pesquisa no Brasil, que aborda as DCNTs. ${ }^{7}$ Quanto aos Objetivos do Desenvolvimento Sustentável, ele se enquadra no Objetivo 3: assegurar uma vida saudável e promover o bem-estar para todas e todos, em todas as idades. ${ }^{8}$

Dessa forma, a questão de pesquisa é: quais são os fatores de risco cardiovascular e qual é a percepção da qualidade de vida dos estudantes de enfermagem de uma universidade federal do interior do Rio Grande do Sul (RS)? Tem-se como objetivo identificar os fatores de risco cardiovascular e a percepção da qualidade de vida entre estudantes de enfermagem de uma universidade federal do RS, Brasil.

\section{Método}


Fatores de risco cardiovascular e qualidade de vida de estudantes de enfermagem $\mid 4$

Estudo transversal descritivo, realizado com estudantes do curso de enfermagem de uma universidade pública localizada no interior do RS. O curso pesquisado iniciou em 1976, possui 4.190 horas, em turno integral e, após recente reforma curricular, passou de oito para 10 períodos. ${ }^{9}$

Adotou-se como critério de inclusão estar regularmente matriculado no curso e, como critérios de exclusão, ser menor de 18 anos, estar gestante, em licença de saúde ou maternidade. No período das coletas, 182 discentes estavam regularmente matriculados. Procedeu-se ao cálculo amostral: ${ }^{10}$

$$
n=\frac{N \cdot \delta^{2} \cdot Z_{\frac{a}{2}}^{2}}{(N-1) E^{2}+\delta^{2} \cdot Z_{\frac{a}{2}}^{2}}
$$

Em que:

$\mathrm{n}=$ tamanho da amostra;

$\mathrm{Z} \alpha / 2=$ valor crítico para o grau de confiança desejado;

$\delta=$ desvio padrão populacional da variável;

$\mathrm{E}=$ erro padrão;

$\mathrm{N}=$ tamanho da população (finita).

Adotaram-se intervalo de confiança de $95 \%$, proporção de $50 \%$ e margem de erro de $5 \%$, resultando em amostra mínima de 124 participantes. Os dados foram coletados entre março e maio de 2018, por meio de: Roteiro de caracterização demográfica e socioeconômica; Formulário de identificação de FRCVs; e Questionário The World Health Organization Quality of Life, bref (WHOQOL-BREF) - versão português. ${ }^{11}$

O roteiro de caracterização demográfica e socioeconômica foi elaborado pelos pesquisadores, para identificar as seguintes variáveis: data de nascimento, sexo, etnia, religião, estado civil, possuir filhos ou não, número de filhos, trabalho/ocupação, semestre do curso, possuir bolsa, renda familiar em salários mínimos (equivalente a $\mathrm{R} \$ 937,00$ na época), distância 
5 | Wickert DC, Silva LMC, Munhoz OL, Schimith MD, Magnago TSBS, Silveira VN

de moradia até a universidade $(\mathrm{km})$, tempo de deslocamento até a universidade (ida e volta), tempo de estudo diário (horas/dia), frequência de estudo à noite (vezes por semana).

O formulário de identificação de FRCVs foi elaborado pelos pesquisadores, tendo como base o Escore de Risco Global de Framingham. ${ }^{12}$ As variáveis elencadas no presente estudo foram: peso e altura (utilizados para o cálculo do Índice de Massa Corporal - IMC), circunferência abdominal, circunferência da cintura e do quadril (relação cintura-quadril), pressão arterial, uso de métodos contraceptivos (anticoncepcional hormonal, dispositivo intrauterino), tabagismo, realização de tratamento prévio e/ou estar realizando tratamento para depressão no momento da coleta, estresse, prática de atividade física, indicadores de baixo/intermediário risco cardiovascular (idade maior que 65 anos, sexo masculino, tabagismo, hipertensão ou préeclâmpsia, obesidade ou obesidade central, sedentarismo, história de evento cardiovascular prematuro em familiares de $1^{\circ}$ grau, história de doença renal, manifestações de aterosclerose, diagnóstico de dislipidemia e diagnóstico de ovário policístico) e indicadores de alto risco cardiovascular (acidente vascular encefálico, infarto agudo do miocárdio, lesão de órgão-alvo, ataque isquêmico transitório, hipertrofia do ventrículo esquerdo, nefropatia, retinopatia, insuficiência cardíaca congestiva de etiologia isquêmica, aneurisma de aorta abdominal, estenose de carótida sintomática, DM, doença vascular periférica, angina no peito e doença renal crônica).

Os FRCVs podem ser baixos/intermediários ou altos. Os estudantes que apresentaram apenas um fator de risco baixo/intermediário foram considerados de baixo risco cardiovascular. Os que apresentavam ao menos um fator de alto risco cardiovascular foram considerados com alto risco para desenvolver doenças cardiovasculares em 10 anos; já os estudantes que apresentavam mais de um fator de risco baixo/intermediário deveriam ser submetidos a exames complementares para o cálculo da probabilidade de desenvolver doença cardiovascular em 10 anos, o que foram orientados a fazer. ${ }^{12}$ Destaca-se que, a partir das variáveis elencadas, consegue-se determinar a quantidade de 
Fatores de risco cardiovascular e qualidade de vida de estudantes de enfermagem |6

FRCVs presentes na população pesquisada, mas não é possível estratificar o risco cardiovascular, devido à ausência de financiamento para realização de exames complementares.

O WHOQOL-BREF é um instrumento de avaliação da QV da OMS, organizado por meio de uma escala do tipo Likert de cinco pontos, variando de intensidade (nada-extremamente), capacidade (nada-completamente), frequência (nunca-sempre) e avaliação (muito insatisfeitomuito satisfeito e muito ruim-muito bom), traduzido e validado para a realidade brasileira, com boa aplicabilidade na população saudável. Possui 26 questões, sendo 24 correspondentes às facetas do WHOQOL-100 e duas relacionadas à QV em geral. Os domínios do WHOQOL-BREF e as respectivas questões são: físico $(3,4,10,15,16,17$ e 18), psicológico (5, 6, 7, 11, 19 e 26), relações sociais $(20,21$ e 22), meio ambiente $(8,9,12,13,14,23,24$ e 25$)$ e QV geral (1 e 2). As questões 3, 4 e 26 são reversas, ou seja, são apresentadas na forma negativa. ${ }^{11}$

Quanto ao processo de obtenção dos dados, os instrumentos foram autoaplicados, exceto as medidas antropométricas, verificadas pelos coletadores, seguindo as recomendações padronizadas, sempre com os mesmos equipamentos, devidamente calibrados, com o intuito de garantir a fidedignidade dos resultados. ${ }^{12-13}$ Destaca-se que os coletadores passaram por capacitação prévia. O teste piloto ocorreu com três discentes, que foram excluídos da pesquisa.

Realizou-se o convite para participação na pesquisa por meio de agendamento prévio com os docentes, tendo em vista que todo o processo de coleta ocorreu em horário de aula. Desta forma, após explicação acerca do projeto, leitura do Termo de Consentimento Livre e Esclarecido (TCLE) e sanar as dúvidas, os estudantes foram convidados a participar do estudo. A pesquisadora responsável assinou o Termo de Confidencialidade e os estudantes que aceitaram participar assinaram o TCLE em duas vias, seguindo os preceitos éticos para pesquisas com seres humanos. Em seguida, realizou-se a coleta dos dados. Ainda, aqueles que não estiveram presentes na oportunidade foram convidados a participar em outro momento, previamente agendado. 
Os dados foram processados por meio de dupla digitação independente no software Microsoft Excel. A seguir, ocorreu-se a conferência das informações, para verificar e corrigir possíveis inconsistências da digitação. Os dados foram analisados por técnicas de estatística descritiva no software Statistical Package for the Social Sciences (SPSS) $18.0^{\varpi}$, respeitando a sintaxe para o cálculo dos resultados do WHOQOL-BREF, ${ }^{11}$ o qual apresentou boa consistência interna $(\alpha=0,80)$, analisada pelo Alfa de Cronbach.

A pesquisa foi conduzida de acordo com os padrões éticos vigentes. Destaca-se que esta foi aprovada por um Comitê de Ética em Pesquisa, sob o Parecer no 2.451.568, em 20/12/2017.

\section{Resultados}

Participaram da pesquisa 131 (75,3\%) estudantes de enfermagem matriculados no curso, sendo $14(10,7 \%)$ do primeiro semestre, $22(16,8 \%)$ do segundo, $21(16,0 \%)$ do terceiro, $19(14,5 \%)$ do quarto, $14(10,7 \%)$ do quinto, $16(12,2 \%)$ do sexto, $12(9,2 \%)$ do sétimo e $13(9,9 \%)$ do oitavo semestre. Salienta-se que durante a coleta de dados não havia turmas de $9^{\circ}$ e $10^{\circ}$ semestres, motivo pelo qual justifica-se a não inclusão desses na amostra do presente estudo. A idade média foi de 22,3 anos $( \pm 4,5)$, com mínimo de 18 e máximo de 50 anos. Na Tabela 1 , serão abordadas variáveis demográficas e socioeconômicas.

Tabela 1- Descrição das variáveis demográficas e socioeconômicas dos estudantes de enfermagem. Rio Grande do Sul, 2018.

\begin{tabular}{|c|c|c|c|}
\hline Variável & $\mathbf{N}$ & $\mathbf{N}$ & $\%$ \\
\hline Sexo & 131 & & \\
\hline Feminino & & 114 & 87,0 \\
\hline Masculino & & 17 & 13,0 \\
\hline Etnia & 131 & & \\
\hline Brancos & & 88 & 67,2 \\
\hline Pardos & & 23 & 17,6 \\
\hline Negros & & 16 & 12,2 \\
\hline
\end{tabular}


Fatores de risco cardiovascular e qualidade de vida de estudantes de enfermagem $\mid 8$

\begin{tabular}{|c|c|c|c|}
\hline Indígenas & & 4 & 3,0 \\
\hline Religião & 127 & & \\
\hline Católica & & 54 & 42,5 \\
\hline Católica e espírita & & 3 & 2,4 \\
\hline Católica e umbanda & & 1 & 0,8 \\
\hline Espírita & & 19 & 15,0 \\
\hline Umbanda & & 4 & 3,1 \\
\hline Evangélica & & 24 & 18,9 \\
\hline Ateu & & 9 & 7,1 \\
\hline Outra* $^{*}$ & & 12 & 9,4 \\
\hline Judaica & & 1 & 0,8 \\
\hline Estado civil & 131 & & \\
\hline Solteiro & & 110 & 84,0 \\
\hline União estável há mais de 6 meses & & 9 & 6,9 \\
\hline Casado & & 7 & 5,3 \\
\hline Separado/desquitado/divorciado & & 2 & 1,5 \\
\hline Outro & & 3 & 2,3 \\
\hline Maternidade/Paternidade & 131 & & \\
\hline $\operatorname{Sim}$ & & 13 & 9,9 \\
\hline Não & & 118 & 90,1 \\
\hline Número de filhos & 13 & & \\
\hline Um filho & & 7 & 53,8 \\
\hline Dois filhos & & 4 & 30,8 \\
\hline Três filhos & & 2 & 15,4 \\
\hline $\begin{array}{l}\text { Distância de moradia até a } \\
\text { universidade }(\mathrm{km})\end{array}$ & 128 & & \\
\hline 0 a 5 & & 52 & 40,6 \\
\hline 5,1 a 10 & & 7 & 5,5 \\
\hline 10,1 a 15 & & 52 & 40,6 \\
\hline 15,1 a 20 & & 17 & 13,3 \\
\hline Trabalho & 130 & & \\
\hline Sim & & 9 & 6,9 \\
\hline Não & & 121 & 93,1 \\
\hline Ocupação & 9 & & \\
\hline Técnico de enfermagem & & 4 & 44,4 \\
\hline Outras & & 5 & 55,6 \\
\hline Bolsa de estudos & 131 & & \\
\hline $\operatorname{Sim}$ & & 61 & 46,6 \\
\hline Não & & 70 & 53,4 \\
\hline
\end{tabular}

* Mórmon, luterana e muçulmana e agnóstica. 
Em relação à renda familiar em salários mínimos, identificou-se a média de 4,7 salários mínimos $( \pm 3,4)$. Ao tratar do tempo de deslocamento para a instituição de ensino em minutos, os participantes dispendiam, em média, $64,5\left(\Perp_{-49,2)}\right.$. O tempo de estudo diário foi em média de 8,1 horas/dia $( \pm 3,8)$. Quanto à frequência de estudo à noite, obteve-se uma média de $3,5( \pm 1,6)$ vezes por semana. Em média os pesquisados apresentaram $68,2 \mathrm{~kg}( \pm 17)$ e um índice de massa corporal (IMC) de $25( \pm 5,5)$. Na Tabela 2, apresenta-se a distribuição do IMC, conforme classificação da OMS. ${ }^{13}$

Tabela 2- Distribuição do IMC de estudantes de enfermagem. Rio Grande do Sul, 2018.

\begin{tabular}{lllcc}
\hline \multicolumn{1}{c}{ IMC } & \multicolumn{1}{c}{ Classificação } & \multicolumn{1}{c}{ Risco de doença } & N & \% \\
\hline$<18,5$ & Magro (baixo peso) & Normal ou Elevado & 12 & 9,1 \\
$18,5-24,9$ & Normal (eutrófico) & Normal & 65 & 49,6 \\
$25-29,9$ & Sobrepeso (pré-obeso) & Pouco elevado & 28 & 21,4 \\
$30-34,9$ & Obesidade grau I & Elevado & 17 & 13,0 \\
$35-39,9$ & Obesidade grau II & Muito elevado & 8 & 6,1 \\
$\geq 40,0$ & Obesidade grau III & Muitíssimo elevado & 1 & 0,8 \\
\hline
\end{tabular}

Em relação às medidas de circunferência abdominal, 92 (70,2\%) estavam dentro dos valores indicados e $39(29,8 \%)$ tinham obesidade central $(86,6 \mathrm{~cm}$ e $\pm 13,6)$. Ainda, quanto à relação cintura-quadril (RCQ), 102 (77,9\%) estudantes apresentaram valores normais e 29 (22,1\%), valores que representam FRCV. Quanto à aferição da Pressão Arterial (PA), 69 (52,7\%) estavam com PA $\leq 120 / 80$ mmHg, e 62 (47,3\%), com PA >120/80 mmHg. A média da Pressão Arterial Sistólica foi de 119 mmHg $( \pm 14,8)$, e a da Pressão Arterial Diastólica, 76 mmHg $( \pm 10,5)$.

Sobre o uso de métodos contraceptivos, das 114 pesquisadas, 80 (70,2\%) referiram fazer uso e, destas, 78 (97,5\%) utilizavam anticoncepcional hormonal e duas (2,5\%) usavam Dispositivo Intrauterino (DIU) de cobre. Dentre os métodos contraceptivos hormonais utilizados, o mais comum foi oral com pausa, utilizado por $53(66,3 \%)$, sendo $23(28,8 \%)$ as que usavam oral contínuo, uma $(1,3 \%)$ que usava injetável mensal e uma $(1,3 \%)$ que usava DIU hormonal. Quanto ao tempo de uso do método anticoncepcional em meses, registrou-se média de 52,3 meses 
Fatores de risco cardiovascular e qualidade de vida de estudantes de enfermagem $\mid 10$

$( \pm 33,4)$. Ainda, verificou-se que 29 das 78 estudantes que utilizavam o anticoncepcional hormonal possuíam algum FRCV, sendo 17 (58,6\%) com obesidade, cinco (17,2\%) com dislipidemia, três (10,3\%) com obesidade e dislipidemia, uma (3,5\%) tabagista, uma $(3,5 \%)$ tabagista e obesa, uma (3,5\%) obesidade e DM e, uma (3,5\%) apresentava somente DM.

Quanto à depressão, $35(26,7 \%)$ referiram ter feito tratamento para a doença em algum momento da vida. Ainda, 21 (16\%) estavam realizando tratamento para depressão no momento da coleta, em média havia 19,3 meses $( \pm 17,4)$. Na questão relacionada ao estresse, $90(68,7 \%)$ se autorreferiram estressados. Quanto à prática de atividade física, 69 (52,7\%) informaram não praticar, $30(22,9 \%)$ disseram praticar menos de 30 minutos em cinco dias da semana e $32(24,4 \%)$ referiram praticar mais de 30 minutos em cinco dias da semana.

Ainda, identificou-se a presença dos FRCVs, classificados em baixo/intermediário (com 11 fatores) e alto risco (com 14 fatores), os quais foram avaliados separadamente. Apresenta-se, na Tabela 3, a distribuição dos indicadores de risco baixo/intermediário e alto entre os participantes.

Tabela 3- Distribuição dos indicadores de risco baixo/intermediário e alto entre os estudantes de enfermagem. Rio Grande do Sul, 2018.

\begin{tabular}{|c|c|c|}
\hline Indicadores de risco & $\mathbf{N}$ & $\%$ \\
\hline \multicolumn{3}{|l|}{ Baixo/intermediário } \\
\hline Idade $>65$ anos & 0 & 0,0 \\
\hline Sexo masculino & 17 & 13,0 \\
\hline Tabagismo & 5 & 3,8 \\
\hline Hipertensão (>140/90 mmHg) ou história de pré-eclâmpsia & 5 & 3,8 \\
\hline $\begin{array}{l}\text { Obesidade (IMC }>30 \mathrm{~kg} / \mathrm{m}^{2} \text { ) ou obesidade central (cintura abdominal medida } \\
\text { na altura do umbigo: }>88 \mathrm{~cm} \text { em mulheres; }>102 \mathrm{~cm} \text { em homens) }\end{array}$ & 41 & 31,3 \\
\hline Sedentarismo & 74 & 56,5 \\
\hline $\begin{array}{l}\text { História de evento cardiovascular prematuro em familiares de } 1^{\circ} \text { grau: pai, } \\
\text { mãe, irmãos (homens }<55 \text { anos e mulheres }<65 \text { anos) }\end{array}$ & 25 & 19,1 \\
\hline História familiar de doença renal (para risco de insuficiência renal) & 16 & 12,2 \\
\hline $\begin{array}{l}\text { Manifestações de aterosclerose: sopros arteriais carotídeos, diminuição ou } \\
\text { ausência de pulsos periféricos }\end{array}$ & 8 & 6,1 \\
\hline Diagnóstico prévio de dislipidemia & 8 & 6,1 \\
\hline Diagnóstico prévio de síndrome de ovário policístico & 19 & 14,5 \\
\hline
\end{tabular}




\begin{tabular}{lll}
\hline Indicadores de alto risco & & \\
Lesão periférica - lesão de órgão-alvo (LOA) & 1 & 0,8 \\
Diabetes mellitus & 3 & 2,3 \\
Doença vascular periférica & 1 & 0,8 \\
Angina no peito & 2 & 1,5 \\
Doença renal crônica & 1 & 0,8 \\
\hline
\end{tabular}

Quanto ao número de fatores de risco baixo/intermediários identificados, 18 (13,7\%) estudantes não apresentaram; $44(33,6 \%)$ apresentaram um fator; 45 (34,4\%), dois fatores; 17 (13\%), três fatores; três $(2,3 \%)$, quatro fatores; e três $(2,3 \%)$, cinco fatores. Destaque para um participante $(0,8 \%)$ que apresentou seis fatores. De forma geral, cada estudante apresentou em média $1,93( \pm 1,0)$ FRCV. Quanto à avaliação da QV, a pontuação média dos domínios foi: domínio físico $61,8( \pm 14,2)$, o psicológico $58,9( \pm 14,7)$, as relações sociais $70,1( \pm 17,0)$, o meio ambiente 57,8 $( \pm 13,8)$ e a autoavaliação da QV 62,9 $( \pm 16,8)$. Apresenta-se, na Tabela 4, a média dos escores da QV, segundo o domínio e semestre do curso.

Tabela 4 - Média e variabilidade dos escores da qualidade de vida, segundo o domínio e semestre do curso. Rio Grande do Sul, 2018.

\begin{tabular}{|c|c|c|c|c|c|c|}
\hline \multirow[b]{2}{*}{$\begin{array}{l}\text { Semestre } \\
\text { do curso }\end{array}$} & \multirow[b]{2}{*}{$\mathbf{N}$} & \multicolumn{4}{|c|}{ Domínios da Qualidade de Vida } & \multirow{2}{*}{$\begin{array}{c}\text { Autoavaliaçãc } \\
\text { da QV }\end{array}$} \\
\hline & & Físico & Psicológico & $\begin{array}{c}\text { Relações } \\
\text { sociais }\end{array}$ & $\begin{array}{c}\text { Meio } \\
\text { ambiente }\end{array}$ & \\
\hline Primeiro & 14 & $62,2 \pm( \pm 10,6)$ & $64,0(+10,6)$ & $72,6( \pm 10,1)$ & $56,8( \pm 9,2)$ & $62,5( \pm 12,7)$ \\
\hline Segundo & 22 & $62,6( \pm 14,3)$ & $61,7( \pm 12,7)$ & $70,8( \pm 12,3)$ & $65,2(+9,4)$ & $64,5(+15,2)$ \\
\hline Terceiro & 21 & $64,8( \pm 16,1)$ & $63,3( \pm 19,6)$ & $73,6( \pm 22,8)$ & $60,3( \pm 14,4)$ & $63,6( \pm 19,9)$ \\
\hline Quarto & 19 & $56,1(+15,6)$ & $53,1( \pm 14,3)$ & $65,8( \pm 17,1)$ & $54,8( \pm 14,8)$ & $56,3( \pm 13,8)$ \\
\hline Quinto & 14 & $58,4( \pm 14,6)$ & $51,7(+14,1)$ & $63,7(+17,5)$ & $50,9(+18,5)$ & $55,3(+16,6)$ \\
\hline Sexto & 16 & $65,6(+12,9)$ & $60,4( \pm 10,1)$ & $72,9( \pm 18,1)$ & $56,0( \pm 12,4)$ & $61,7( \pm 16,8)$ \\
\hline Sétimo & 12 & $64,9( \pm 13,9)$ & $61,8( \pm 11,4)$ & $74,7(+13,4)$ & $55,2( \pm 11,9)$ & $62,5( \pm 18,9)$ \\
\hline Oitavo & 13 & $57,7( \pm 12,9)$ & $54,2( \pm 17,9)$ & $66,0( \pm 20,3)$ & $57,8( \pm 15,1)$ & $58,3( \pm 19,8)$ \\
\hline
\end{tabular}

As médias dos escores das 26 questões (facetas) do WHOQOL-BREF são apresentadas na Figura 1, separadas por domínio. Destacam-se as três questões negativas do questionário, que são precedidas com sinal de asterisco, como pode ser observado. 
Fatores de risco cardiovascular e qualidade de vida de estudantes de enfermagem $\mid 12$

Figura 1- Média dos escores das questões (facetas) do WHOQOL-BREF. Rio Grande do Sul, 2018.

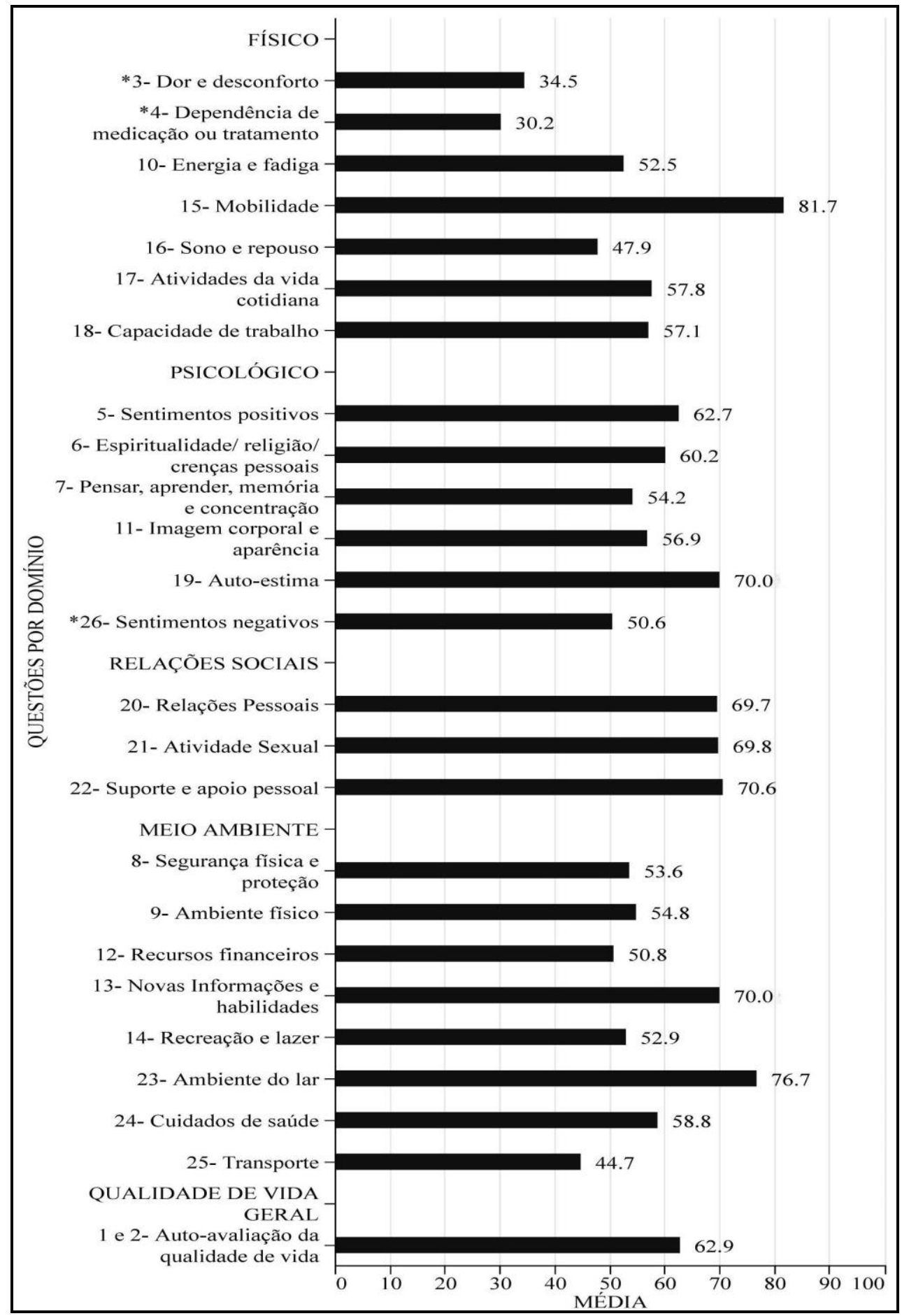

${ }^{*}$ Questões invertidas (escala de Likert de cinco pontos)

A questão com pior percepção foi a relacionada ao meio de transporte, com média de $44,7( \pm 27,8)$. Já a melhor média, $81,7( \pm 18,3)$, está relacionada à capacidade de locomoção. 
13 | Wickert DC, Silva LMC, Munhoz OL, Schimith MD, Magnago TSBS, Silveira VN

\section{Discussão}

O perfil demográfico dos estudantes pesquisados corrobora com a Pesquisa do Perfil da Enfermagem no Brasil de 2013, que identificou predominância de mulheres $(86,2 \%)$, brancos (57,9\%), seguidos por pardos (31,3\%), pretos (6,6\%), amarelos (2,5\%) e indígenas (0,3\%). ${ }^{14}$ Essa informação suscita reflexão, pois, nas origens históricas da profissão, a enfermagem era exercida apenas por mulheres, associando o cuidado ao feminino, o que pode refletir na atual representação da enfermagem, apesar do número de homens estar crescendo na profissão. O resultado requer atenção também no quesito étnico, pois historicamente o acesso ao ensino superior era de pessoas brancas, na sua maioria homens, e com maior poder aquisitivo. Esta realidade vem sendo lentamente revertida com as ações afirmativas, políticas de acesso e permanência nas universidades. ${ }^{15}$

Quanto ao trabalho e atuação profissional, os resultados encontrados assemelham-se a um estudo no qual 97,6\% dos 164 pesquisados não possuíam vínculo empregatício, em especial devido ao fato de o curso ser em período integral. ${ }^{16}$ Sobre a presença de bolsa de estudo, os resultados assemelham-se à Pesquisa do Perfil dos Estudantes do Brasil, a qual constatou que 75,6\% dos estudantes de uma universidade pública não contribuíam para a manutenção da casa. Dos que o faziam, $46,8 \%$ contribuíam trabalhando e $53,2 \%$, por meio de bolsas de estudo. ${ }^{17}$

Referente à renda familiar, estes recebiam, em média, $\mathrm{R} \$ 4.432,01 /$ mês. No período das coletas, o valor mínimo necessário para o brasileiro satisfazer as necessidades básicas previstas era de $\mathrm{R} \$ 3.701,70$ mensais. ${ }^{18}$ Pode-se concluir que, em média, os participantes recebiam salários adequados para satisfazer as necessidades familiares, no entanto, devem-se considerar as particularidades de cada contexto e família.

O tempo gasto com o deslocamento demonstrou média semelhante com pesquisa que identificou que os estudantes de enfermagem de uma universidade pública de São Paulo demoravam mais de 60 minutos no percurso entre sua casa e a universidade. ${ }^{19}$ Considerando o 
Fatores de risco cardiovascular e qualidade de vida de estudantes de enfermagem | 14

Censo de 2010, percebeu-se que os pesquisados gastavam 17,54 minutos a mais com deslocamento diário do que a média da população brasileira residente em cidades do interior, que levam, em média, 46,96 minutos. Ou seja, o tempo é comparável às médias das capitais sem Região Metropolitana (67,01 minutos). ${ }^{20}$ Ressalta-se que a locomobilidade na cidade em questão é deficitária e afeta a QV da população, assim, investir em mobilidade urbana propicia benefícios aos seus moradores, como a melhoria do bem-estar, evitando problemas relacionados ao acesso à saúde, educação e segurança. ${ }^{21}$

Os resultados desta pesquisa, no que se refere ao tempo de estudo à noite, mostram que os estudantes vivenciavam uma extensa carga horária de estudos e, por vezes, isso leva a abandonar as atividades físicas e alimentação saudável, influenciando e aumentando a probabilidade de desenvolver FRCVs. ${ }^{6}$ Estudo qualitativo, realizado na Bahia, trouxe a carga horária de estudos, estágios, atividades extracurriculares, cobranças no rendimento acadêmico e uma consequente alimentação inadequada como não promotoras da QV. ${ }^{22}$

Verificou-se que a média do IMC foi de $25 \mathrm{~kg} / \mathrm{m}^{2}$, valor pertencente à classificação de sobrepeso ou pré-obesidade. Ressalta-se, ainda, que quase $1 / 4$ dos participantes foi identificado com obesidade central, que reflete a gordura visceral e é um dos fatores para síndrome metabólica, sendo os valores de referência $\leq 88 \mathrm{~cm}$ na mulher e $\leq 102 \mathrm{~cm}$ nos homens. ${ }^{23}$ Outra medida verificada nos participantes foi a RCQ, que reflete a gordura corporal total e possui como valores de referência: para homens $\leq 0,9$, e para mulheres, $\leq 0,8 ; 22,1 \%$ dos pesquisados apresentaram valores que representam risco cardiovascular. ${ }^{13}$ Com o panorama e o contexto nos quais os estudantes estão inseridos, pode-se refletir que a carga de estudos, com consequente a falta de horários para lazer e prática de exercícios, pode estar contribuindo com o quadro geral de saúde e QV, refletindo diretamente nos FRCVs.

Relacionado à PA, $62(47,3 \%)$ pesquisados apresentaram valores acima de 120/80 mmHg. Para a Sociedade Brasileira de Cardiologia, a classificação da PA de acordo com a medição 
15 | Wickert DC, Silva LMC, Munhoz OL, Schimith MD, Magnago TSBS, Silveira VN

casual ou no consultório, a partir de 18 anos de idade, é considerada normal nos valores igual ou menor que 120/80 mmHg, de 121-139/81-89 mmHg é classificada como pré-hipertensão, acima disto, considera-se como hipertensão. ${ }^{12}$ Ressalta-se que os valores de uma medição isolada não fazem diagnóstico da HAS. Os participantes com valores de PA alterados foram orientados a procurar a unidade de saúde de referência. Estudo que avaliou o risco cardiovascular de acadêmicos de enfermagem encontrou a média da PA sistólica de 106,8 mmHg e PA diastólica de 71,6 mmHg, ${ }^{6}$ assemelhando-se aos resultados encontrados.

Pesquisa realizada com 21.074 brasileiras, em idade entre 18 e 49 anos, mostrou que 51,5\% utilizavam algum contraceptivo hormonal, sendo o mais utilizado o contraceptivo oral $(33,8 \%)$. O presente estudo identificou que as estudantes de enfermagem vinham utilizando mais contraceptivos hormonais que a média das brasileiras, realidade que pode ser explicada devido às possibilidades estudadas durante o curso. Em contrapartida, identificou-se um número considerável delas que possuíam FRCVs (tabagismo, obesidade, DM, dislipidemia) e utilizavam anticoncepcionais hormonais, uma forma arriscada de contracepção. Pesquisa traz que alguns FRCVs, como HAS, tabagismo, dislipidemia, obesidade e DM, quando presentes, restringem as indicações ao uso de contraceptivos hormonais, devido a potenciais complicações, como trombose e acidente vascular encefálico. ${ }^{24}$

Dentre os pesquisados, $90(68,7 \%)$ se consideravam estressados. Pesquisa trouxe o estresse como prejudicial à $\mathrm{QV}$, sendo consideradas geradoras de estresse entre discentes de enfermagem a sobrecarga de atividades, a distância diária percorrida entre a residência e a universidade e a falta de tempo para o lazer. ${ }^{19}$ Ainda, estudo identificou a presença de estresse médio em 74,5\% dos estudantes de enfermagem de quatro instituições de ensino superior, e estresse elevado entre $15,1 \%$ deles. ${ }^{25}$ Isso demonstra uma realidade preocupante e provoca a reflexão sobre a saúde física e mental durante o período da graduação, também visualizado no que tange à depressão entre os universitários. O processo depressivo pode levar a um maior 
Fatores de risco cardiovascular e qualidade de vida de estudantes de enfermagem | 16

estresse, em especial devido a seu impacto negativo no cotidiano, o que pode resultar em diferentes disfunções que impactam na vida como um todo, inclusive na área acadêmica. A OMS traz relação entre depressão e saúde física, tendo como exemplo as DCVs, que podem levar à depressão, sendo possível também ocorrer o contrário. ${ }^{26}$

Ainda, a maioria dos estudantes referiu não praticar atividade física (52,7\%), e o valor encontrado é semelhante à média nacional brasileira, visto que uma pesquisa identificou que $62,1 \%$ dos brasileiros são sedentários. ${ }^{27}$ Pode-se inferir que o elevado sedentarismo entre os pesquisados esteja relacionado à competitividade do mercado de trabalho, carga horária de estudos e estágios, além de possíveis barreiras pessoais, como falta de motivação, de dinheiro e companhia para a prática de atividades físicas, sendo esta atribuída como um significado de QV em estudo. ${ }^{19}$

Um ponto importante a ser observado é que os dois FRCVs com maior frequência eram modificáveis (sedentarismo, obesidade ou obesidade central). A avaliação dos FRCVs não deve ser vista somente como um mero cálculo, mas, sim, como um meio de promover reflexões entre discentes, docentes e gestores da universidade onde a pesquisa foi desenvolvida, tendo em vista a existência de um comportamento de risco nesse cenário. Destaca-se que a prevenção dos riscos cardiovasculares desde a juventude é fundamental para que o processo de envelhecimento ocorra com saúde e QV. ${ }^{6}$

Pesquisa realizada com estudantes de enfermagem da Bahia inferiu que 64,3\% dos participantes não praticavam atividades físicas e 35,7\% dispunham de pouco tempo para o lazer. $^{22}$ Outra pesquisa obteve que $84(67,9 \%)$ acadêmicos de enfermagem estavam insuficientemente ativos e 17,8\%, sedentários. Ainda, 36,9\% estavam com a circunferência abdominal acima dos valores adequados, ou seja, apresentavam obesidade central. Traz, ainda, que a prática da atividade física e a alimentação são comportamentos de grande influência no desenvolvimento de DCNTs. ${ }^{6}$

Os resultados evidenciados com o WHOQOL-BREF apontaram uma pior percepção na QV no domínio meio ambiente, seguido pelo domínio psicológico, e o domínio com melhor 
avaliação foi o das relações sociais, seguido pelo físico. No que tange à autoavaliação da QV, a maioria dos participantes avaliou sua QV como boa ou muito boa, e estava satisfeita ou muito satisfeita com sua saúde.

Esses resultados assemelham-se a um estudo realizado com 116 estudantes de enfermagem do Amazonas, que obteve um escore de 71,2 para o domínio das relações sociais, 65,7 no domínio psicológico, 58,6 no domínio meio ambiente e 57,4 para o domínio físico. Notase que, além de escores semelhantes, obteve-se a melhor avaliação da QV no mesmo domínio, o das relações sociais, que se refere às questões de apoio social e vida sexual. Este resultado é muito importante para os docentes, pois pode indicar a necessidade de ações junto aos estudantes, visto que muitas vezes suas relações sociais são modificadas quando eles vêm de outras localidades para cursar a graduação. ${ }^{28}$

Um estudo mostrou que, na percepção dos discentes de enfermagem de uma universidade pública, a amizade entre os colegas, as práticas esportivas e a possibilidade de obtenção de bolsas de iniciação científica ou de trabalho favorecem a QV. Entre os fatores que diminuem a QV, figuram a distância entre o local de moradia e a universidade, o curso em turno integral, a sobrecarga de atividades, os gastos financeiros e a falta de tempo para lazer. ${ }^{19}$

Já o domínio meio ambiente, que apresentou escores mais baixos, está relacionado às questões sobre segurança, clima, barulho, poluição, questões financeiras, lazer, acesso aos serviços de saúde, transporte e moradia. No RS, em 2017, foram registrados 3.316 homicídios, 1.460 estupros e 1.893 óbitos em acidentes de transporte..$^{29}$

Avaliando os estudantes segundo o semestre do curso, percebeu-se que o quinto semestre possuía a pior avaliação da QV nos domínios psicológico, meio ambiente, relações sociais e autoavaliação da QV. Uma das hipóteses para a baixa QV no quinto semestre pode ser o início das aulas práticas no âmbito hospitalar, avaliado em estudos como uma circunstância não promotora da QV. ${ }^{19,28}$ Ainda, de acordo com o atual currículo do curso de enfermagem da 
Fatores de risco cardiovascular e qualidade de vida de estudantes de enfermagem $\mid 18$

referida instituição, o quinto semestre é o com maior carga horária, 480 horas, seguido pelo quarto semestre, com 450 horas, ${ }^{9}$ sendo a extensa carga horária já discutida anteriormente como um aspecto que compromete a percepção acerca da QV. ${ }^{22}$

Por fim, quanto aos melhores escores da QV, o segundo semestre obteve a melhor percepção nos domínios meio ambiente e autoavaliação da QV. Pode-se cogitar tal resultado no sentido de que os estudantes do segundo semestre haviam tido um semestre anterior de adaptação à turma e à instituição, e ainda não estavam inseridos nas aulas práticas nos serviços de saúde, o que pode repercutir em menor nível de exigência e menor estresse. ${ }^{22}$

O estudo foi realizado em uma realidade específica, objetivando a identificação dos FRCVs e da percepção da QV e, por isso, há limitação na generalização dos achados. Ainda, são imprescindíveis outras abordagens que façam associações e abordem a subjetividade humana, a fim de suprir as lacunas existentes e promover maior atenção às questões de saúde dos estudantes de enfermagem.

\section{Conclusão}

O perfil predominante dos estudantes de enfermagem da instituição investigada é de mulheres, brancas, solteiras, sem filhos, católicas e sem ocupação profissional. Evidenciou-se alta prevalência dos FRCVs sedentarismo e obesidade entre os participantes, tornando evidente uma séria e desafiadora realidade. No quesito QV, identificou-se que a maior problemática se relaciona ao meio ambiente e questões psicológicas e, em contrapartida, tem-se a melhor percepção da QV nas relações sociais e nas questões físicas.

Diante dos resultados, faz-se necessária uma articulação intersetorial para a realização de ações voltadas à promoção da QV e minimização da presença dos FRCVs entre os estudantes, pois os mais frequentes são modificáveis. Pesquisar a presença dos FRCVs e a QV entre estudantes de enfermagem permite ampliar o olhar para as diferentes esferas de gestão 
envolvidas no apoio a esta parcela da sociedade. Tal fato representa um avanço no que se relaciona a pensar nessa população, que necessita de um suporte que transcenda o foco no processo formativo e parta para um conjunto de reflexões e ações voltadas ao desenvolvimento como sujeito e futuro profissional enfermeiro.

\section{Referências}

1. Organização Pan-Americana de Saúde (OPAS-BR). Doenças cardiovasculares [Internet]. Brasília (DF): OPAS-BR; 2017 [acesso em 2019 fev 12]. Disponível em: https://www.paho.org/bra/index.php?option=com_content\&view=article\&id=5253:doencascardiovasculares\&Itemid $=1096$

2. Malta DC, Andrade SSCA, Oliveira TP, Moura L, Prado RR, Souza MFM. Probabilidade de morte prematura por doenças crônicas não transmissíveis, Brasil e regiões, projeções para 2025. Rev Bras Epidemiol. 2019;22:e190030. doi: 10.1590/1980-549720190030

3. World Health Organization (WHO). WHOQOL: measuring quality of life [Internet]. Geneva: WHO; 1997 [cited $2019 \quad$ Feb 12$]. \quad$ Available from: https://apps.who.int/iris/bitstream/handle/10665/63482/WHO_MSA_MNH_PSF_97.4.pdf?sequence=1\&isA llowed=y

4. Hickel F, Fabro BR, Bertoldi EG. Fatores de risco cardiovascular e qualidade de vida de acadêmicos de Medicina da Universidade Federal de Pelotas. Rev AMRIGS. 2015 [acesso em 2018 out 19];59(3):186-91. Disponível em: https://livrozilla.com/doc/744346/05_1498_revista-amrigs.indd

5. Cruz-Sánchez E, Orosio-Méndez M, Cruz-Ramírez T, Bernardino-Garcia A, Vásquez-Domínguez L, Galindo-Palma N, et al. Factores de riesgo cardiovascular en estudiantes de enfermería de una universidad pública. Enferm Univ. 2016;13(4):226-32. doi: 10.1016/j.reu.2016.09.004

6. Santos JS, Patrício ACFA, Alves KLA, Albuquerque KF, Pereira IL, Félix IVB. Avaliação para riscos cardiovasculares em estudantes de enfermagem. REME Rev Min Enferm. 2015;19(4):848-53. doi: $10.5935 / 1415-2762.20150065$

7. Ministério da Saúde (BR), Secretaria de Ciência, Tecnologia e Insumos Estratégicos, Departamento de Ciência e Tecnologia. Agenda de prioridades de pesquisa do Ministério da Saúde - APPMS [Internet]. Brasília (DF): Ministério da Saúde; 2018 [acesso em 2020 mar 06]. Disponível em: https://bvsms.saude.gov.br/bvs/publicacoes/agenda_prioridades_pesquisa_ms.pdf

8. United Nations (UN). Transforming our world: the 2030 agenda for sustainable development [Internet]; 2015 [cited 2019 Sept $18]$. Available from: 
Fatores de risco cardiovascular e qualidade de vida de estudantes de enfermagem $\mid 20$

https://sustainabledevelopment.un.org/content/documents/21252030\%20Agenda\%20for\%20Sustainable\%2 0Development\%20web.pdf

9. Universidade Federal de Santa Maria (UFSM). Portal do ementário. Ementa do curso de Enfermagem [Internet]. 2017 [acesso em 2019 mar 14]. Disponível em: https://portal.ufsm.br/ementario/curso.html?idCurso=762

10. Callegari-Jacques SM. Bioestatística: princípios e aplicações. Porto Alegre: Artmed; 2018. 255 p.

11. Universidade Federal do Rio Grande do Sul (UFRGS), Grupo Whoqol. Projeto Whoqol-Bref [Internet]. 2016 [acesso em 2020 jul 15]. Disponível em: https://www.ufrgs.br/qualidep/qualidade-de-vida/projeto-whoqol-bref

12. Précoma DB, Oliveira GMM, Simão AF, Dutra OP, Coelho OR, Izar MCO, et al. Atualização da diretriz de prevenção cardiovascular da Sociedade Brasileira de Cardiologia - 2019. Arq Bras Cardiol. 2019;113(4):787-891. doi: 10.5935/abc.20190204

13. Associação Brasileira para o Estudo da Obesidade e da Síndrome Metabólica (ABESO). Diretrizes brasileiras de obesidade [Internet]. 2016 [acesso em 2019 jun 12]. Disponível em: https://abeso.org.br/wpcontent/uploads/2019/12/Diretrizes-Download-Diretrizes-Brasileiras-de-Obesidade-2016.pdf

14. Conselho Federal de Enfermagem (COFEN). Pesquisa Perfil da Enfermagem no Brasil [Internet]. 2016 [acesso em 2018 jun 12]. Disponível em: http://www.cofen.gov.br/perfilenfermagem/index.html

15. Silva GHG. Um panorama das ações afirmativas em Universidades Federais do Sudeste brasileiro. Cad Pesqui. 2019;49(173):184-206. doi: 10.1590/198053145665

16. Garcia AKA, Moraes A, Guariente MHDM. Perfil de estudantes ingressantes de um curso de Enfermagem do Sul do Brasil: caracterização dos hábitos de leitura e estudo. Semina Cienc Biol Saude. 2016;37(2):47-54. doi: 10.5433/1679-0367.2016v37n2p47

17. Universidade do Estado do Rio de Janeiro (UERJ), Instituto de Medicina Social (IMS), Rede Observatório de Recursos Humanos em Saúde. Alunos de graduação em Enfermagem - perfil, expectativas e perspectivas profissionais: relatório [Internet]. 2008 [acesso em 2018 jun 12]. Disponível em: http://www.obsnetims.org.br/uploaded/30_4_2013__0_Relatorio_Pesquisa_Alunos_Graduacao.pdf

18. Departamento Intersindical de Estatística e Estudos Socioeconômicos (DIEESE). Pesquisa Nacional da Cesta Básica de Alimentos. São Paulo; 2018 [acesso em 2019 jun 12]. Disponível em: https://www.dieese.org.br/analisecestabasica/salarioMinimo.html\#2017

19. Oliveira BM, Mininel VA, Felli VEA. Qualidade de vida de graduandos de enfermagem. Rev Bras Enferm. 2011;64(1):130-5. doi: 10.1590/S0034-71672011000100019

20. Instituto Brasileiro de Geografia e Estatística (IBGE). Pesquisa Nacional por Amostra de Domicílios: um panorama da saúde no Brasil. 2010 [acesso em 2017 out 22]. Disponível em: 
http://bvsms.saude.gov.br/bvs/publicacoes/pnad_panorama_saude_brasil.pdf

21. Lopes AFA, Guerra MEA. O programa cidade sustentável, seus indicadores e metas: instrumentos metodológicos para a avaliação da sustentabilidade no município de Prata/MG. In: Pacheco JTR, Kawanishi JY, Nascimento R. Meio ambiente e desenvolvimento sustentável. Ponta Grossa (PR): Atena; 2019. Cap. 12; p. 139-56.

22. Freitas AMC, Bárbara JFRS, Vale PRLF, Carvalho SS, Carvalho LF, Nery GS. Percepções de estudantes de enfermagem referente à qualidade de vida na trajetória acadêmica. Rev Enferm UFSM. 2017;7(2):152-66. doi: 10.5902/2179769225391

23. Sociedade Brasileira de Endocrinologia e Metabologia. Síndrome Metabólica [Internet]. 2017 [acesso em 2018 jun 12]. Disponível em: https://www.endocrino.org.br/sindrome-metabolica/

24. Corrêa DAS, Felisbino-Mendes MS, Mendes MS, Malta DC, Velasquez-Melendez G. Fatores associados ao uso contraindicado de contraceptivos orais no Brasil. Rev Saúde Pública. 2017;51(1):1-10. doi: 10.1590/S1518-8787.2017051006113

25. Bublitz S, Guido LA, Lopes LFD, Freitas EO. Associação entre estresse e características sociodemográficas e acadêmicas de estudantes de enfermagem. Texto Contexto Enferm. 2016; 25(4):e2440015. doi: 10.1590/0104-07072016002440015

26. Organização Pan-Americana de Saúde (OPAS-BR). Folha Informativa- depressão [Internet]. 2018 [acesso em 2019 fev 15]. Disponível em: https://www.paho.org/bra/index.php?option=com_content\&view=article\&id=5635:folha-informativadepressao\&Itemid=1095

27. Instituto Brasileiro de Geografia e Estatística (IBGE). Pesquisa Nacional por Amostra de Domicílios: prática de esporte e atividade física - 2015 [Internet]. 2017 [acesso em 2019 jun 12]. Disponível em: https://biblioteca.ibge.gov.br/visualizacao/livros/liv100364.pdf

28. Gama ASM. Qualidade de vida de estudantes de enfermagem do Amazonas, Brasil. Rev Baiana Enferm. 2016;30(4):1-9. doi: 10.18471/rbe.v30i4.17011

29. Instituto de Pesquisa Econômica Aplicada (IPEA). Atlas da violência [Internet]. 2017 [acesso em 14 jul 2020]. Disponível em: https://www.ipea.gov.br/atlasviolencia/filtros-series

Editor Científico Chefe: Cristiane Cardoso de Paula

Editor Associado: Alexa Pupiara Flores Coelho

Fomento / Agradecimento: Universidade Federal de Santa Maria 
Fatores de risco cardiovascular e qualidade de vida de estudantes de enfermagem | 22

\section{Autor correspondente}

Daiana Cristina Wickert

E-mail: daianacristinaw@gmail.com

Endereço: Av. Roraima no 1000 - Cidade Universitária

Centro de Ciências da Saúde/PPGENF - Prédio 26

Bairro Camobi- Santa Maria, RS, Brasil

CEP: $97105-900$

\section{Contribuições de Autoria}

\section{1 - Daiana Cristina Wickert}

Concepção ou desenho do estudo/pesquisa, análise e/ou interpretação dos dados, revisão final com participação crítica e intelectual no manuscrito.

\section{2 - Laís Mara Caetano da Silva}

Concepção ou desenho do estudo/pesquisa, análise e/ou interpretação dos dados, revisão final com participação crítica e intelectual no manuscrito.

\section{3 - Oclaris Lopes Munhoz}

Análise e/ou interpretação dos dados, revisão final com participação crítica e intelectual no manuscrito.

\section{4 - Maria Denise Schimith}

Revisão final com participação crítica e intelectual no manuscrito.

\section{5 - Tânia Solange Bosi de Souza Magnago}

Análise e/ou interpretação dos dados, revisão final com participação crítica e intelectual no manuscrito.

\section{6 - Vanessa do Nascimento Silveira}

Concepção ou desenho do estudo/pesquisa, análise e/ou interpretação dos dados, revisão final com participação crítica e intelectual no manuscrito.

\section{Como citar este artigo}

Wickert DC, Silva LMC, Munhoz OL, Schimith MD, Magnago TSBS, Silveira VN. Fatores de risco cardiovascular e qualidade de vida de estudantes de enfermagem. Rev. Enferm. UFSM. 2021 [Acesso em: Anos Mês Dia]; vol.11 e5: 122. DOI:https://doi.org/10.5902/2179769243038 